\title{
Erratum to: Updates on Clostridium difficile in Europe
}

Paola Mastrantonio and Maja Rupnik

\section{Erratum to:}

Book "Updates on Clostridium difficile in Europe" in: P. Mastrantonio and M. Rupnik, Adv Exp Med Biol - Advances in Microbiology, Infectious Diseases and Public Health, https://doi.org/10.1007/978-3-319-72799-8

Due to a different technical process the source lines were set differently in the original version and have now been corrected.

\footnotetext{
The original online version for these chapters can be found at https://doi.org/10.1007/978-3-319-72799-8_1 https://doi.org/10.1007/978-3-319-72799-8_2 https://doi.org/10.1007/978-3-319-72799-8_3 https://doi.org/10.1007/978-3-319-72799-8_4 https://doi.org/10.1007/978-3-319-72799-8_5 https://doi.org/10.1007/978-3-319-72799-8_6 https://doi.org/10.1007/978-3-319-72799-8_7 https://doi.org/10.1007/978-3-319-72799-8_8 https://doi.org/10.1007/978-3-319-72799-8_9 https://doi.org/10.1007/978-3-319-72799-8_10 https://doi.org/10.1007/978-3-319-72799-8_11 https://doi.org/10.1007/978-3-319-72799-8_12 https://doi.org/10.1007/978-3-319-72799-8_13 https://doi.org/10.1007/978-3-319-72799-8_14 https://doi.org/10.1007/978-3-319-72799-8
} 